\title{
MEK-Inhibition beim inoperablen NRAS-mutierten Melanom
}

\section{Hier steht eine Anzeige.}

\author{
Wenn eine Immuntherapie bei fortgeschrittenem \\ inoperablem NRAS-mutiertem malignem Melanom \\ versagt, ist guter Rat teuer. In einer Phase-III-Studie wurden \\ die Effizienz und Sicherheit des MEK-Inhibitors Binimetinib \\ im Vergleich zu Dacarbazin getestet.
}

\section{Springer}

$V^{i}$ iele maligne Melanome haben genetische Alterationen wie BRAF-, NRAS- oder NF1-Mutationen. Aktivierte NRAS-Mutationen etwa liegen bei mehr als $20 \%$ der metastasierten Melanome vor. Die Therapieoptionen für NRAS-mutierte Melanome jenseits der Immuntherapien sind derzeit noch begrenzt. Präklinische in-vitro-Studien lassen vermuten, dass BRAF-mutierte und NRASmutierte Melanome sensibel auf MEK-Inhibition reagieren.

In einer randomisierten, multizentrischen Phase-III-Studie wurde nun überprüft, wie effizient und sicher die MEK-Inhibition mit Binimetinib im Vergleich zu Dacarbazin bei Patienten mit fortgeschrittenem inoperablem NRAS-mutiertem Melanom im Stadium IIIC oder IV ist. Die 402 Teilnehmer waren zuvor unbehandelt oder hatten während oder nach vorangegangener Immuntherapie einen Progress erlitten. Sie wurden auf entweder Binimetinib (45 mg oral zweimal täglich; $n=296)$ oder Dacarbazin (1.000 $\mathrm{mg} / \mathrm{m}^{2}$ i. v. alle drei Wochen; $\mathrm{n}=133$ ) randomisiert. Primärer Endpunkt war das progressionsfreie Überleben in der Intention-totreat-Population.

Das mediane Follow-up betrug 1,7 Monate. Das mediane progressionsfreie Überleben lag in der Binimetinib-Gruppe bei 2,8 Monaten, in der Dacarbazin-Gruppe bei 1,5 Monaten $(\mathrm{p}<0,001)$. Das mediane Gesamtüberleben wurde nach 228 Todesfällen (161 unter Binimetinib, 67 unter Dacarbazin) analysiert und lag unter Binimetinib bei 11 Monaten (95\%-Konfidenzintervall [95\%-KI] $8,9-13,6)$, in der Dacarbazin-Gruppe bei 10,1 Monaten (95\%-KI $7,0-16,5)(p=0,50)$. Unter Binimetinib sprachen anteilmäßig mehr Patienten auf die Therapie an als unter Dacarbazin.

Nebenwirkungen vom Grad 3-4 traten bei mindestens 5\% der Patienten der Sicherheitspopulation auf: Erhöhte Kreatinkinase bei 52 (19\%) von 269 Patienten der Binimetinibgruppe versus keiner von 114 Patienten der Dacarbazingruppe, Hypertonie bei 20 (7\%) versus zwei (2\%), Anämie bei fünf (2\%) versus sechs (5\%) und Neutropenie bei zwei (1\%) versus zehn (9\%). Schwerwiegende Nebenwirkungen aller Grade traten bei 91 (34\%) Patienten der Binimetinibgruppe und 25 (22\%) Patienten der Dacarbazingruppe auf.

Fazit: Im Vergleich zu Dacarbazin verlängert Binimetinib das progressionsfreie Überleben signifikant. Der Wirkstoff sei weitgehend verträglich, so die Autoren. Möglicherweise könne er eine neue Therapieoption für die Behandlung von Patienten mit fortgeschrittenem inoperablem NRAS-mutiertem Melanom nach Versagen einer Immuntherapie sein.

Kathrin von Kieseritzky

Dummer $\mathrm{R}$ et al. Binimetinib versus dacarbazine in patients with advanced NRAS-mutant melanoma (NEMO): a multicentre, open-label, randomised, phase 3 trial. Lancet Oncol 2017; 18: 435-45 